\title{
Splicing factor 2/alternative splicing factor contributes to extracellular signal-regulated kinase activation in hepatocellular carcinoma cells
}

\author{
YAWEI ZHAO ${ }^{1,2^{*}}$, TING ZHU $^{2 *}$, XUEYING ZHANG ${ }^{2}$, QINGYANG WANG ${ }^{2}$, \\ JIYAN ZHANG ${ }^{2}$, WENBIN JI ${ }^{3}$ and YUANFANG MA ${ }^{1}$ \\ ${ }^{1}$ Laboratory of Cellular and Molecular Immunology, Henan University, Kaifeng, Henan 475001; \\ ${ }^{2}$ Department of Molecular Immunology, Institute of Basic Medical Sciences, Beijing 100850; \\ ${ }^{3}$ Department of Hepatobiliary Surgery, The Chinese PLA General Hospital, Beijing 100853, P.R. China
}

Received May 29, 2014; Accepted March 5, 2015

DOI: $10.3892 / \mathrm{mmr} .2015 .3851$

\begin{abstract}
The splicing factor is important in cancer development, modulation numerous tumor suppressors and oncogenes, and regulation of multiple signaling pathways. Splicing factor 2/alternative splicing factor (SF2/ASF) is a proto-oncogene, which has been implicated in the development of hepatocellular carcinoma. However, the underlying molecular mechanism remains to be elucidated. In the present study, it was identified that SF2 knockdown had no effect on tumor necrosis factor (TNF)- $\alpha$-induced activation of the c-Jun N-terminal protein kinase (JNK) pathway, the p38 pathway, or the IKK pathway in hepatoma cell lines. However, SF2 knockdown led to reduced levels of basal ERK activation and TNF- $\alpha$-induced ERK activation, without changing the protein levels of ERK. Consequently, SF2 knockdown marginally enhanced TNF- $\alpha$-induced cell death. Furthermore, SF2 knockdown and blockade of ERK activation partially suppressed TNF- $\alpha$-induced interleukin- 6 expression. As SF2 knockdown exhibited no role in basal Akt activation and serum-induced Akt activation, it is unlikely that SF2 affects ERK activation through modulating the protein levels of certain growth factor receptors. In conclusion, the data suggest that SF2 contributes
\end{abstract}

Correspondence to: Dr Wenbin Ji, Department of Hepatobiliary Surgery, The Chinese PLA General Hospital, 28 Fuxing Street, Beijing 100853, P.R. China

E-mail: jiwenbin1999@126.com

Dr Yuanfang Ma, Laboratory of Cellular and Molecular Immunology, Henan University, 28 Jinming Street, Kaifeng, Henan 475001, P.R. China

E-mail: mayf@henu.edu.cn

${ }^{*}$ Contributed equally

Key words: splicing factor 2/alternative splicing factor, extracellular signal-regulated kinase, tumor necrosis factor- $\alpha$, interleukin-6, hepatocellular carcinoma to the elevated levels of ERK activation in hepatocellular carcinoma cells through modulating key component(s) downstream of growth factor receptors and upstream of ERK.

\section{Introduction}

Hepatocellular carcinoma (HCC) is one of the most common types of cancer, ranked the third most common cause of cancer-related mortality worldwide, particularly in Africa and Asia (1). Extracellular signal-regulated kinase (ERK), a member of the mitogen-activated protein kinases (MAPK) superfamily, which also includes c-Jun $\mathrm{N}$-terminal protein kinase (JNK), and p38 family of kinases, has been implicated in HCC development (1). ERK is activated by a variety of extracellular stimuli, from growth factors, such as epidermal growth factor (EGF), to proinflammatory cytokines, including tumor necrosis factor (TNF)- $\alpha(2,3)$. Growth factors tend to simultaneously activate the ERK and Akt pathways (2), whereas TNF- $\alpha$ activates all three major groups of MAPKs, as well as the I $\mathrm{B}$ kinase (IKK) pathway $(3,4)$. Extensive studies have shown that chronic inflammation associated with persistent viral infections and/or persistent exposure to hepatotoxic agents is clearly the primary inducer of HCC $(1,5)$. TNF- $\alpha$ and interleukin (IL)- 6 are key proinflammatory cytokines involved in HCC development $(6,7)$. ERK protects against TNF- $\alpha$-induced apoptosis and mediates TNF- $\alpha$-induced IL-6 expression $(8,9)$. However, the mechanisms underlying the aberrant activation of the ERK pathway in HCC remain largely unclear.

Alternative splicing modulates the expression of various oncogene and tumor-suppressor isoforms (10-12). Mutations in components of the spliceosome were recently identified in several types of cancer and are predicted to be driver mutations, supporting the concept that splicing factors are important in cancer development $(13,14)$. Splicing factor 2/alternative splicing factor (SF2/ASF) is a member of the arginine/serine-rich splicing factor family and has been identified as a proto-oncogene that is amplified in human tumors and can transform immortalized mouse fibroblasts, which form sarcomas in nude mice (15). Recently, it has been 
proposed that SF2/ASF is protumorigenic in HCC through increased alternative splicing and consequent inactivation of Krüppel-like factor 6 (KLF6), a zinc finger transcription factor that inhibits cellular growth in part by transcriptional activation of p21 $(16,17)$. However, it remains unknown whether SF2/ ASF also employs other mechanism(s) to contribute to HCC development.

The current study was undertaken to investigate the mechanism(s) other than KLF6 inactivation by which SF2/ASF contributes to the development of HCC.

\section{Materials and methods}

Cell culture and transfection. Cells (SMMC-7721 and BEL-7402) were purchased from the Shanghai Institutes for Biological Sciences (Shanghai, China) and were cultured in Dulbecco's modified Eagle's medium supplemented with $10 \%$ fetal bovine serum (Hyclone, Logan, UT, USA), $100 \mathrm{U} / \mathrm{ml}$ penicillin and $100 \mu \mathrm{g} / \mathrm{ml}$ streptomycin (Sigma-Aldrich, St. Louis, MO, USA) and were maintained at $37^{\circ} \mathrm{C}$ with $5 \% \mathrm{CO}_{2}$. Small interfering RNAs (siRNAs) against human SF2/ASF (GCATCTACGTGGGTAACTT, GGAGTTTGTACGGAAAGAA) and non-targeting control siRNA were synthesized by Shanghai GenePharma Co., Ltd. (Shanghai, China). Transfection was performed with Lipofectamine 2000 (Invitrogen Life Technologies, Carlsbad, CA, USA) according to the the manufacturer's instructions.

ELISA. Cells were stimulated with or without $10 \mathrm{ng} / \mathrm{ml} \mathrm{TNF}-\alpha$ (R\&D Systems, Minneapolis, MN, USA) for $24 \mathrm{~h}$. Then, interleukin (IL)-6 levels in the supernatants were measured using an ELISA kit (eBioscience, San Diego, CA, USA) according to the manufacturer's instructions.

Immunoblotting analysis. Cells were washed twice with ice-cold phosphate-buffered saline (PBS) and were then lysed with $20 \mathrm{mM}$ Tris/ $\mathrm{HCl}$ (pH 7.6), $250 \mathrm{mM} \mathrm{NaCl}, 3 \mathrm{mM}$ EDTA,3 mM EGTA, 0.5\% NP-40, 1 mM DTT, 5 mM NaF, $2 \mathrm{mM} \mathrm{Na}_{3} \mathrm{VO}_{4}$ and $0.2 \mu \mathrm{M}$ Aprotinin. The whole cell extract was clarified at $4^{\circ} \mathrm{C}$ at $13,800 \mathrm{x} g$ for $15 \mathrm{~min}$. The quantity of protein recovered was quantified with a Bradford protein assay (Invitrogen Life Technologies). Equal quantities of protein were resolved by sodium dodecyl sulfate-polyacrylamide gel electrophoresis (SDS-PAGE) and then transferred to Hybond-P polyvinylidene difluoride (PVDF) membranes (GE Healthcare Life Sciences, Chalfont, UK). Membranes were sequentially incubated with primary antibody overnight at $4^{\circ} \mathrm{C}$ and horseradish peroxidase-conjugated secondary antibody for $1 \mathrm{~h}$ at room temperature. Bound antibody was detected using an enhanced chemiluminescence kit (GE Healthcare Life Sciences) and Kodak X-ray film (Rochester, NY, USA). Antibodies against SF2 (sc-33652), actin (sc-8432), IKK $\alpha / \beta$ (sc-7607) and p38 (sc-535) were purchased from Santa Cruz Biotechnology Inc. (Santa Cruz, CA, USA). Antibodies against phospho-IKK $\alpha / \beta$ (2697S), phospho-JNK (9251S), phospho-p38 (9215S), phospho-ERK (9102S) and ERK (4370S) were obtained from Cell Signaling Technology Inc. (Danvers, MA, USA). Antibodies against JNK (612541) were obtained from BD Biosciences (Franklin Lakes, NJ, USA). Antibodies against phospho-Akt (2118-1) were purchased from Epitomics (Cambridge, MA, USA).
Apoptosis analysis. Cells were adjusted to a density of $2 \times 10^{5}$ cells $/ \mathrm{ml}$, added to 24 -well plates in $0.5 \mathrm{ml}$ per well regular culture medium. Cells were treated with $10 \mathrm{ng} / \mathrm{ml}$ TNF- $\alpha$ and $1 \mu \mathrm{g} / \mathrm{ml}$ cycloheximide (CHX, Sigma-Aldrich) for $24 \mathrm{~h}$. Cells were washed with PBS twice and stained with Annexin V-phycoerythrin and 7-AAD (Nanjing KeyGen Biotech, Nanjing, China) for $15 \mathrm{~min}$ at room temperature in the dark. The level of apoptosis was determined by measuring the fluorescence of the cells with a flow cytometer (FACSCalibur; BD Biosciences).

Statistical analysis. Statistically significant differences between groups were identified using 2-tailed Student's t-test. Statistical analysis was conducte using SPSS software, version 13.0 (SPSS, Inc., Chicago, IL, USA). $\mathrm{P}<0.05$ was considered to indicate a statistically significant difference.

\section{Results}

SF2 knockdown marginally enhanced TNF- $\alpha$-induced cell death in hepatoma cells. In order to explore whether SF2/ASF affects TNF- $\alpha$-induced cell death and TNF- $\alpha$-induced activation of multiple signaling pathways in hepatoma cell lines, two siRNAs were designed against SF2. SMMC-7721 and BEL-7402 hepatoma cells were transfected with small interfering (si)RNA against SF2 or the non-targeting control (NC) siRNA. After (48 h) transfection, cell lysates were obtained and subjected to immunoblotting. As shown in Fig. 1A and B, the SF2 siRNAs designed significantly inhibited SF2 expression, as compared with the non-targeting control siRNA. TNF- $\alpha$ usually does not trigger cell death unless de novo protein synthesis is blocked by reagents, such as CHX (18). Under these conditions, hepatoma cells with or without SF2 knockdown were subjected to TNF- $\alpha / \mathrm{CHX}$ treatment for $24 \mathrm{~h}$. Apoptosis analysis revealed that SF2 knockdown exhibited marginal increase in the cytotoxicity of TNF- $\alpha$ in the two cell lines (Fig. 1C and D).

SF2 knockdown leads to reduced levels of basal ERK activation as well as TNF- $\alpha$-induced ERK activation in hepatoma cells. The study also aimed to investigate whether SF2/ASF affects the cytotoxicity of TNF- $\alpha$ by interfering with TNF- $\alpha$-induced activation of signaling pathways in hepatoma cell lines. For this purpose, SMMC-7721 cells with or without $\mathrm{SF} 2$ knockdown were subjected to TNF- $\alpha$ treatment for $15 \mathrm{~min}$. Immunoblotting analysis revealed that SF2 knockdown had no effect on TNF- $\alpha$-induced activation of the JNK pathway, the p38 pathway or the IKK pathway in hepatoma cells (Fig. 2A). However, SF2 knockdown led to reduced levels of basal ERK activation as well as TNF- $\alpha$-induced ERK activation without changing the protein levels of ERK (Fig. 2A). The effect of SF2 knockdown on ERK activation was also observed in BEL-7402 cells (Fig. 2B). As ERK exhibits a pro-survival role, it is possible that SF2 antagonizes the cytotoxicity of TNF- $\alpha$ by augmenting ERK activity.

SF2 knockdown and blockade of ERK activation suppress TNF- $\alpha$-induced IL- 6 production in hepatoma cells. In addition to TNF- $\alpha$, IL-6 is also a key proinflammatory cytokine involved in HCC development (6). ERK has been demonstrated 
A

\section{SMMC-7721}
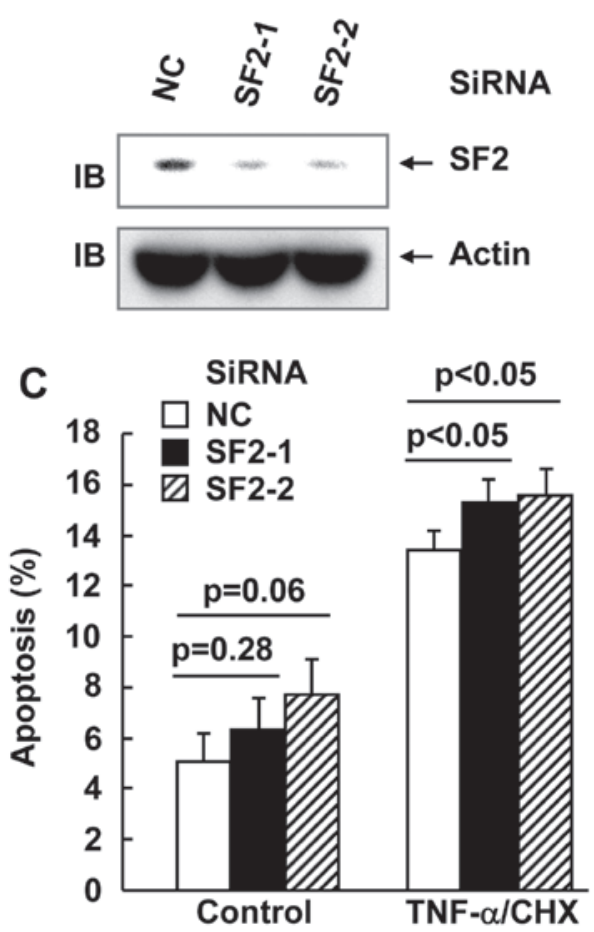

B

\section{BEL-7402}

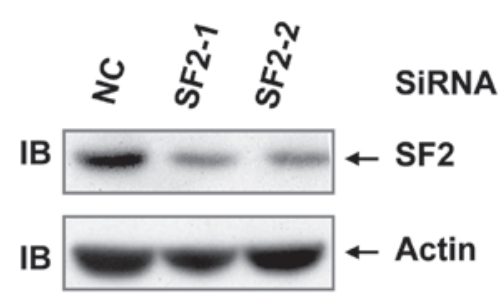

D

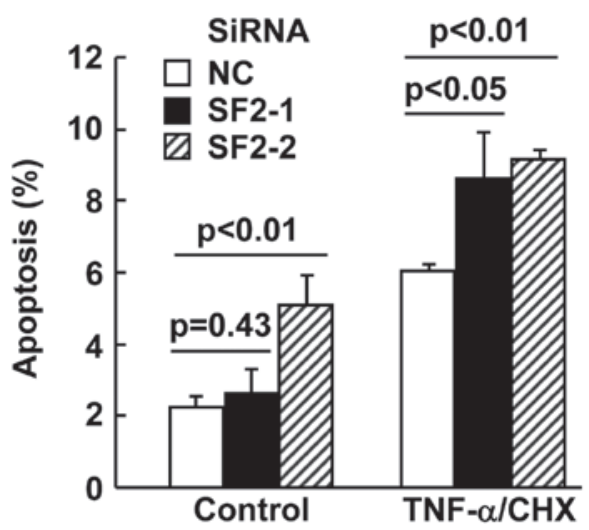

Figure 1. SF2 knockdown enhanced TNF- $\alpha$-induced cell death in hepatoma cells. SMMC-7721 and BEL-7402 hepatoma cells were transfected with siRNA against SF2 or the non-targeting control (NC) siRNA. After transfection (48 h), cell lysates were made from (A) SMMC-7721 cells and (B) BEL-7402 cells and subjected to IB. After the transfection, (C) SMMC-7721 cells and (D) BEL-7402 cells were treated with or without TNF- $\alpha$ (10 ng/ml)/CHX (1 $\mu \mathrm{g} / \mathrm{ml})$ for $24 \mathrm{~h}$. Then cells were subjected to apoptosis analysis. SF2, splicing factor 2 ; TNF- $\alpha$, tumor necrosis factor- $\alpha$; NC, non-targeting control; siRNA, small interfering RNA; IB, immnoblotting;. CHX, cycloheximide.

A

\section{SMMC-7721}

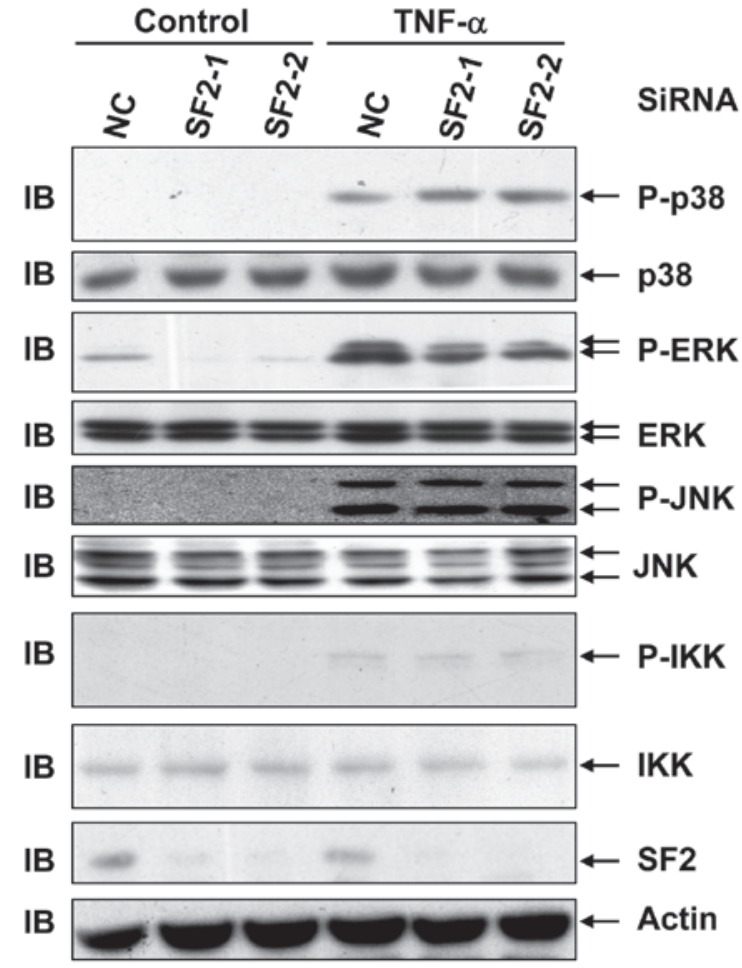

B

\author{
BEL-7402
}

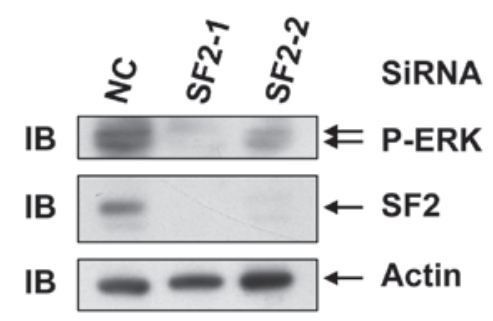

Figure 2. SF2 knockdown leads to reduced levels of basal ERK activation as well as TNF- $\alpha$-induced ERK activation in hepatoma cells. (A) SMMC-7721 and (B) BEL-7402 hepatoma cells were transfected with siRNA against SF2 or the non-targeting control siRNA. After transfection (48 h), cells were treated with or without TNF- $\alpha(10 \mathrm{ng} / \mathrm{ml})$ for $15 \mathrm{~min}$. Then cell lysates were made and subjected to immunoblotting. SF2, splicing factor 2 ; TNF- $\alpha$, tumor necrosis factor- $\alpha$; siRNA, small interfering RNA; NC, non-targeting control; ERK, extracellular-signal regulated kinase; JNK, c-Jun N-terminal protein kinase; IKK, I $\mathrm{B}$ kinase. 

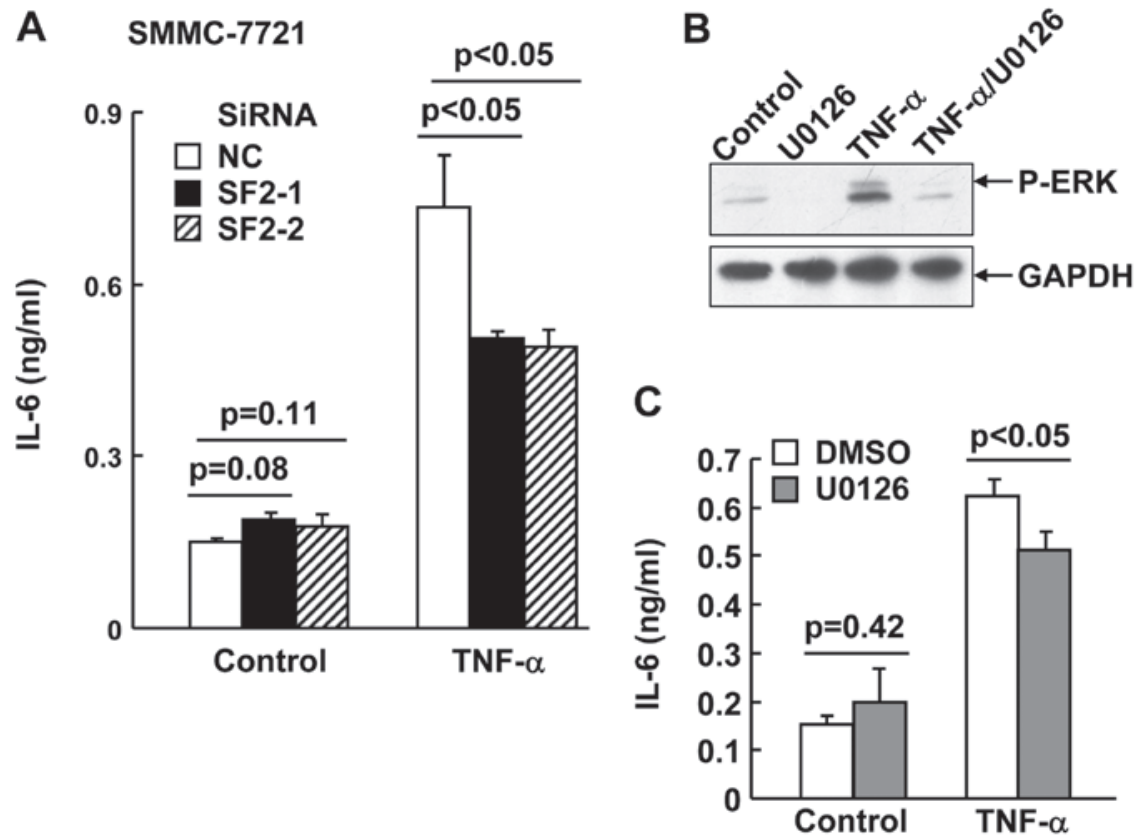

Figure 3. SF2 knockdown and blockade of ERK activation suppresses TNF- $\alpha$-induced IL-6 production in hepatoma cells. (A) SMMC-7721 cells were transfected with siRNA against SF2 or the non-targeting control siRNA. After transfection (48 h), cells were treated with or without TNF- $\alpha$ (10 ng/ml) for $24 \mathrm{~h}$. Then the supernatants were subjected to ELISA. (B) After SMMC-7721 cells were pretreated with U0126 (10 $\mu \mathrm{M})$ or DMSO of equal volume for 30 min, the cells were stimulated with or without TNF- $\alpha(10 \mathrm{ng} / \mathrm{ml})$ for $15 \mathrm{~min}$. Then cell lysates were made and subjected to immunoblotting. (C) After SMMC-7721 cells were pretreated with U0126 $(10 \mu \mathrm{M})$ or DMSO of equal volume for $30 \mathrm{~min}$, the cells were stimulated with or without TNF- $\alpha(10 \mathrm{ng} / \mathrm{ml})$ for $24 \mathrm{~h}$. Then the supernatants were subjected to ELISA. ERK, extracellular signal-regulated kinase; TNF, tumor necrosis factor; IL, interleukin; siRNA, small interfering RNA; SF2, splicing factor 2; DMSO, dimethyl sulfoxide.

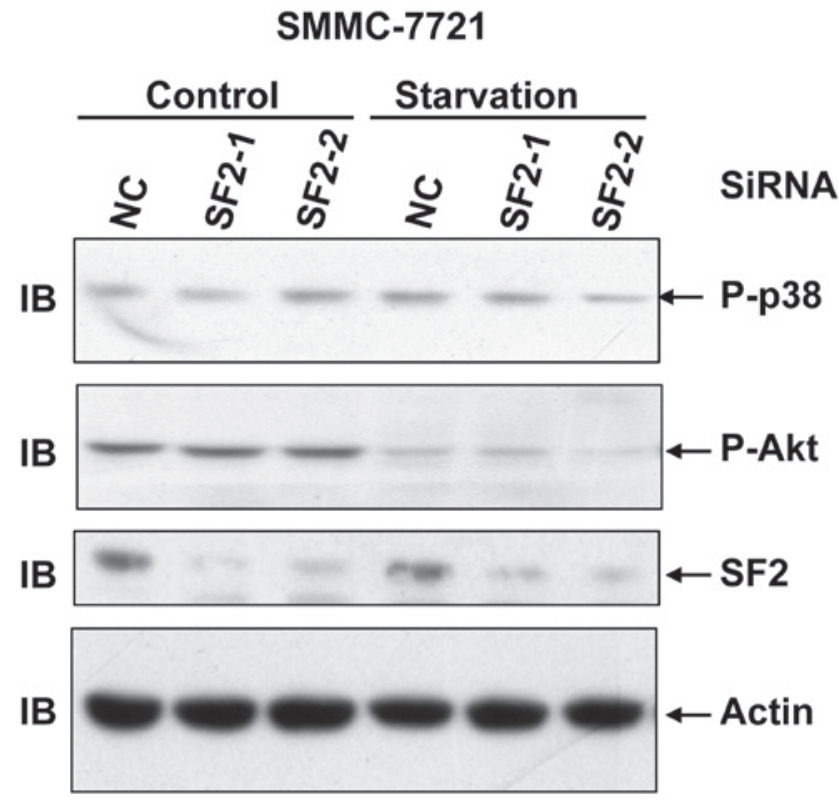

Figure 4. SF2 knockdown exhibits no role in Akt activation in hepatoma cells. SMMC-7721 cells were transfected with siRNA against SF2 or the non-targeting control siRNA. After transfection ( $48 \mathrm{~h}$ ), cells were subjected to serum starvation for $24 \mathrm{~h}$ or left untreated. Then cell lysates were made and subjected to immunoblotting.

to contribute to TNF- $\alpha$-induced IL- 6 production (9). In this scenario, it is of interest to investigate whether SF2 promotes TNF- $\alpha$-induced IL- 6 production. SMMC-7721 cells with or without SF2 knockdown were subjected to TNF- $\alpha$ treatment for $24 \mathrm{~h}$. ELISA analysis with the supernatant revealed that
SF2 knockdown resulted in partially reduced IL-6 production in response to TNF- $\alpha$ (Fig. 3A). Consistently, U0126, a specific inhibitor of the ERK pathway (Fig. 3B), also partially suppressed TNF- $\alpha$-induced IL-6 production in SMMC-7721 cells (Fig. 3C).

SF2 knockdown is not involved in Akt activation in hepatoma cells. ERK is activated not only by TNF- $\alpha$, but also by growth factors. Growth factors tend to simultaneously activate the ERK pathway and the Akt pathway (2). To investigate whether SF2 contributes to ERK activation by affecting the levels of the growth factor receptors, Akt activation with or without serum starvation was conducted as it is known that serum contains various growth factors. As SF2 knockdown exhibited no role in basal Akt activation and serum-induced Akt activation (Fig. 4), it is unlikely that SF2 affects ERK activation through modulating the protein levels of certain growth factor receptors.

\section{Discussion}

Recently, splicing factor oncoprotein SRSF1 has been shown to be a potent proto-oncogene that is upregulated in numerous types of cancer and can transform immortal mouse and human cells $(15,19)$. SF2 is a member of the splicing factor family, which is important in the maintenance of cell growth, proliferation and inflammation (17,20,21). However, the underlying mechanisms remain unclear.

Certain studies show that targeting SF2 may be a strategy for the treatment of leukemia as SF2 silencing promotes the apoptosis of white blood cells $(13,14)$. Whether SF2 exhibits 
the same role in liver cancer cells remains unclear. In the present study, it was demonstrated that SF2 knockdown leads to reduced levels of basal ERK activation, as well as TNF- $\alpha$-induced ERK activation without changing the protein levels of ERK. As SF2 knockdown exhibited no role in basal Akt activation and serum-induced Akt activation, SF2 affects ERK activation through modulating molecular events upstream of ERK, but downstream of the receptors. Consistently, SF2 knockdown only suppresses ERK activation, but not p38/JNK activation in response to TNF- $\alpha$.

ERK may promote HCC development through various mechanisms, including enhancing cell proliferation, cell survival and IL-6 production. Since SF2 contributes to ERK activation in such cells, SF2 knockdown marginally enhanced TNF- $\alpha$-induced cell death and partially suppressed TNF- $\alpha$-induced IL- 6 expression. In conclusion, the present data support the notion that SF2 may be a therapeutic target for the treatment of hepatocellular carcinoma.

\section{References}

1. Llovet JM, Burroughs A and Bruix J: Hepatocellular carcinoma. Lancet 362: 1907-1917, 2003.

2. Worster DT, Schmelzle T, Solimini NL, Lightcap ES, Millard B, Mills GB, Brugge JS and Albeck JG: Akt and ERK control the proliferative response of mammary epithelial cells to the growth factors IGF-1 and EGF through the cell cycle inhibitor p57Kip2. Sci Signal 5: ra19, 2012.

3. Zhang J, Wang Q, Zhu N, Yu M, Shen B, Xiang J and Lin A: Cyclic AMP inhibits JNK activation by CREB-mediated induction of c-FLIP ${ }_{L}$ and MKP-1, thereby antagonizing UV-induced apoptosis. Cell Death Differ 15: 1654-1662, 2008.

4. Yan J, Xiang J, Lin Y, Ma J, Zhang J, Zhang H, Sun J, Danial NN, Liu $J$ and Lin A: Inactivation of BAD by IKK inhibits TNF-induced apoptosis independently of NF- $\mathrm{KB}$ activation. Cell 152: 304-315, 2013.

5. He G and Karin M: NF-kappaB and STAT3 - key players in liver inflammation and cancer. Cell Res 21: 159-168, 2011.

6. Naugler WE, Sakurai T, Kim S, et al: Gender disparity in liver cancer due to sex differences in MyD88-dependent IL-6 production. Science 317: 121-124, 2007.

7. Balkwill F and Coussens LM: Cancer, an inflammatory link. Nature 431: 405-406, 2004
8. Sakurai T, Itoh K, Higashisuji $\mathrm{H}$, Nonoguchi K, Liu Y, Watanabe H, Nakano T, Fukumoto M, Chiba T and Fujita J: Cirp protects against tumor necrosis factor- $\alpha$-induced apoptosis via activation of extracellular signal-regulated kinase. BBA-Mol Cell Res 1763: 290-295, 2005.

9. Suarez-Cuervo C, Harris KW, Kallman L, Vaananen HK and Selander KS: Tumor necrosis factor-alpha induces interleukin-6 production via extracellular-regulated kinase 1 activation in breast cancer cells. Breast Cancer Res Treat 80: 71-78, 2003.

10. Venables JP: Aberrant and alternative splicing in cancer. Cancer Res 64: 7647-7654, 2004.

11. Srebrow A and Kornblihtt AR: The connection between splicing and cancer. J Cell Sci 119: 2635-2641, 2006.

12. Kim E, Goren A and Ast G: Insights into the connection between cancer and alternative splicing. Trends Genet 24: 7-10, 2008.

13. Yoshida K, Sanada M, Shiraishi Y et al: Frequent pathway mutations of splicing machinery in myelodysplasia. Nature 478: 64-69, 2011.

14. Quesada V, Ramsay AJ and Lopez-Otin C: Chronic lymphocytic leukemia with SF3B1 mutation. N Engl J Med 366: 2530, 2012.

15. Karni R, de Stanchina E, Lowe SW, Sinha R, Mu D and Krainer AR: The gene encoding the splicing factor SF2/ASF is a proto-oncogene. Nat Struct Mol Biol 14: 185-193, 2007.

16. Yea S, Narla G, Zhao X, Garg R, Tal-Kremer S, Hod E, Villanueva A, Loke J, Tarocchi M, Akita K, Shirasawa S, Sasazuki T, Martignetti JA, Llovet JM and Friedman SL: Ras promotes growth by alternative splicing-mediated inactivation of the KLF6 tumor suppressor in hepatocellular carcinoma. Gastroenterology 134: 1521-1531, 2008.

17. Muñoz Ú, Puche JE, Hannivoort R, Lang UE, Cohen-Naftaly M and Friedman SL: Hepatocyte growth factor enhances alternative splicing of the Kruppel-like factor 6 (KLF6) tumor suppressor to promote growth through SRSF1. Mol Cancer Res 10: 1216-1227, 2012.

18. Tang G, Minemoto Y, Dibling B, Purcell NH, Li Z, Karin M and Lin A: Inhibition of JNK activation through NF- $\kappa B$ target genes. Nature 414: 313-317, 2001.

19. Anczuków O, Rosenberg AZ, Akerman M, Das S, Zhan L, Karni R, Muthuswamy SK and Krainer AR: The splicing factor SRSF1 regulates apoptosis and proliferation to promote mammary epithelial cell transformation. Nat Struct Mol Biol 19: 220-228, 2012.

20. Geuking MB, Cahenzli J, Lawson MA, Ng DC, Slack E, Hapfelmeier S, McCoy KD and Macpherson AJ: Intestinal bacterial colonization induces mutualistic regulatory $\mathrm{T}$ cell responses. Immunity 34: 794-806, 2011.

21. Panasyuk G, Nemazanyy I, Zhyvoloup A, Filonenko V, Davies D, Robson M, Pedley RB, Waterfield M and Gout I: mTORbeta splicing isoform promotes cell proliferation and tumorigenesis. J Biol Chem 284: 30807-30814, 2009. 